\title{
Multimodal Image Fusion for Brain Image Based on Nonsubsampled Shearlets Transform
}

\author{
Na Zhang ${ }^{1, \text { a) }}$, Peiguang Wang ${ }^{2, \text { b) }}$ \\ ${ }^{1}$ College of Physics Science \& Technology, Hebei University, Baoding, 071000, China. \\ ${ }^{2}$ college of Electronic \& Information Engineering, Hebei University, Baoding, 071000, China. \\ a) snna2work@163.com \\ b) Corresponding author: 1034867982@qq.com
}

\begin{abstract}
Multi-modal medical image fusion has been emerging as a new and promising research area due to the increasing demands in clinical application. A novel fusion rule for medical brain image fusion is proposed in this paper based on the Nonsubsampled Shear lets Transform (NSST). The functional image is converted into HIS color space for betteredcorrelation of intensity channel from chromatic channels. The high frequency sub-bands are fused by using fusion rule based on edge feature measurement. The low frequency sub-bands are combined by using fusion rule based on information and clarity measurement. The performance of the proposed fusion method is assessed by experiment, and the results indicate the proposed method outperforms the traditional approaches.
\end{abstract}

Key words: Medical Image Fusion; Nonsubsampled Shear Let’s Transform; HIS Transform.

\section{INTRODUCTION}

The image fusion is the process of combing information from two or more sensed on acquired images into a single composite image that is more informative and more suitable for visual perception and computer or visual processing [1]. The objective is to reduce uncertainty, minimize redundancy in the output, and maximize relevant information pertaining to an application or a task. Image fusion plays a vital role in the field of biomedical image analysis. Due to different tomography theories, the various medical image modalities are developed [2]. There are several popular imaging modalities such as Computer Tomography (CT) scan and MRI (Magnetic Resonance Imaging) scan, Single Positron Emission Tomography (SPECT) and Positron Emission Tomography (PET) scans. The anatomical imaging modalities, such as CT, MRI, provide structural detail useful for perfect localization and delineation of lesions. However, functional imaging modalities, like PET or SPECT, provide metabolic information by which lesion can be classified into begin and malignant. This functional information also helps in perfectly evaluating the tumor extent or invasion into adjacent tissues. Hence, by fusion of functional image with an anatomical image, it is possible to provide structural correlation to functional information which gives additional diagnostic information [3].

In the color space, there is an advantage in the possibility of presenting the data in different color channels, including hue (H, dominant wavelength), intensity (I), and saturation ( $\mathrm{S}$, called HIS approach). The red, green, and blue (RGB) image is divided into spatial (I) and spectral (H and S) information. There are two kinds of possible transformations: (1) transform to three images (channels) presented in RGB into I, H, and S directly, and (2) separate the color channels unto average brightness representing the surface roughness (intensity), dominant wavelength (hue), and purity (saturation). The HIS approach has become standard procedure in image analysis. It has the ability to enhance the color of highly correlated data and can fuse disparate data.

In this paper, a novel fusion rules for brain image fusion based on NSST is proposed, which gives the simulation results and the performance evaluation. The paper is organized as follows. In section 2 the Nonsubsampled shear lets transform algorithm and HIS transform is introduced. The image fusion framework based on NSSTT is presented in 
section 3. In section 4 the performance of the proposed fusion method is evaluated via experiments. Finally, the conclusion follows.

\section{BASIC THEORY}

\section{Nonsubsampled Shearlets Transform}

The Nonsubsampled shear lets Transform can decompose the image into infinite high frequency sub-bands by controlling the parameter. Hence it has much higher oriented sensitivity and can capture more geometrical feature of the image than other multi-resolution analysis tools. The NSST can deal multidimensional and multidirectional signals effectively, especially with distributed discontinuities such as edge. The NSST is a kind of multi-scale and multidirection analysis framework of the image processing which has been proved to be the best directional image representation.

It can be divided into two phases: multi-scale factorization is analyzed by Nonsubsampled Palladian pyramid (NSLP), and multi-direction factorization is realized by the shearing filter. The NSLP is used to decompose the image into low and high frequency components. Several high frequency sub-bands are obtained by the direction shearing filters. More theoretical details of NSST can be found in [4].

Let $\mathrm{C}_{\mathrm{Hx}}^{\mathrm{l}, \theta}(\mathrm{i}, \mathrm{j})$ and $\mathrm{C}_{\mathrm{Lx}}(\mathrm{i}, \mathrm{j})$ denote the high and low frequency component separately.

$$
\operatorname{NSST}(x)=\left\{C_{H x}^{l, \theta}(i, j), C_{L x}(i, j)\right\}, x=A, B
$$

Where the $A, B$ is represented the medical brain image.

\section{HIS Color Model}

In the linear HIS color model, a multispectral image or panchromatic image with red, green and blue channels to hue $(\mathrm{H})$, intensity $(\mathrm{I})$ and saturation $(\mathrm{S})$ components [5]. The hue is the property of the spectral wavelength, the intensity shows the brightness, and the saturation is the purity of the spectrum. The RGB to HIS conversion is defined by transformation:

$$
\left[\begin{array}{c}
I \\
v 1 \\
v 2
\end{array}\right]=\left[\begin{array}{ccc}
1 / 3 & 1 / 3 & 1 / 3 \\
-\sqrt{2} / 6 & -\sqrt{2} / 6 & 2 \sqrt{2} / 6 \\
1 / \sqrt{2} & -1 / \sqrt{2} & 6
\end{array}\right]\left[\begin{array}{l}
R \\
G \\
B
\end{array}\right]
$$

Hue and saturation are obtained from $v 1$ and $v 2$ :

$$
\begin{gathered}
H=\tan ^{-1}(v 1 / v 2) \\
S=\sqrt{v 1^{2}+v 2^{2}}
\end{gathered}
$$
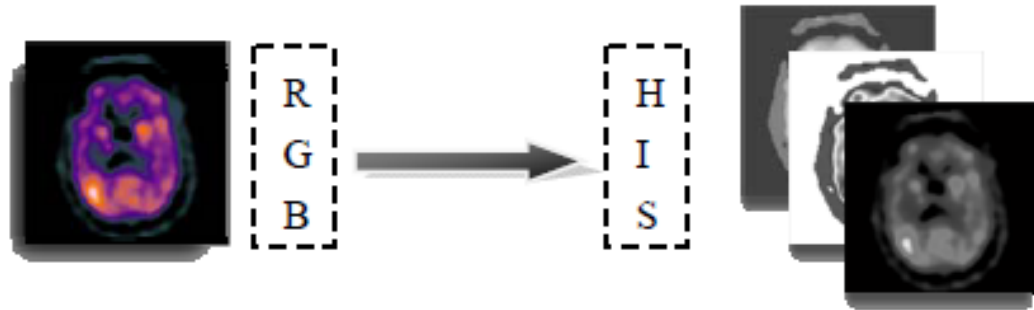

FIGURE 1. The example of the RGB to HIS color space. 


\section{MULTI-MODAL MEDICAL IMAGE FUSION FRAME}

The image fusion scheme based on Nonsubsampled shear lets Transform (NSST) and HIS can be described as follows:

Step 1: NSST decomposition

The source image is decomposed into low frequency components, and multi-direction high frequency components via Nonsubsampled shear lets Transform respectively. Meanwhile the SPECT image which represented in RGB format is converted to the HIS color space. Then the low frequency and high frequency coefficients are fused by different fusion rules respectively.

Step 2: Low frequency coefficients fusion

In order to obtain more information, the low frequency coefficients of the fused image are combined by the measurement according to the entropy and clarity measurement in proposed algorithm. Consider low frequency coefficients of the source images $A$ and $B$ as $C_{L A}(i, j)$ and $C_{L B}(i, j)$ respectively.

Let $F\left(C_{L X}(i, j)\right)$ denotes the region saliency feature by the $m \times n$ sliding widow which located at $(i, j)$. And the fusion rule can be described as Eq. 5:

$$
C_{L F}(I, J)= \begin{cases}C_{L A}(i, j), & F\left(C_{L A}(i, j)\right)>F\left(C_{L B}(i, j)\right) \\ C_{L B}(i, j), & F\left(C_{L A}(i, j)\right) \leq F\left(C_{L B}(i, j)\right)\end{cases}
$$

Where $\mathrm{F}\left(\mathrm{C}_{\mathrm{LX}}(\mathrm{i}, \mathrm{j})\right)$ is the feature measurement value of the region energy and clarity of the input images by the following Eq. 6 ?

$$
F\left(C_{L X}(i, j)\right)=\max \left|\sum_{i=1}^{m} \sum_{j=1}^{n} p(i, j) \log p(i, j)+\sum_{i=1}^{m} \sum_{j=1}^{n} \sqrt{\frac{\left(C_{L X}(i, j)-C_{L X}(i, j+1)\right)^{2}+\left(C_{L X}(i, j)-C_{L X}(i+1, j)\right)^{2}}{2}}\right|
$$

Step 3: High frequency coefficients fusion

The high frequency sub-bands are the representation of edge information of an image. Therefore, the fusion rule of high frequency coefficients can be obtained as in Eq. 7:

$$
C_{H F}=W(i, j) \times C_{H A}^{l, \theta}(i, j)+\left[(1-W(i, j)) \times C_{H B}^{l, \theta}(i, j)\right]
$$

Where the $W(i, j)$ is the weight value of the edge feature measurement [6], which describes as followed:

$$
W(i, j)=\sum_{i=1}^{m} \sum_{j=1}^{n} P(i, j) \log \frac{P(i, j)}{P_{A}(i, j) P_{B}(i, j)}
$$

Where the $\mathrm{P}(\mathrm{i}, \mathrm{j})$ is the normalization union grey level histogram of the source image.

Step 4: Inverse NSST reconstruction

Using the fused low and high frequency coefficients, the fused image is obtained by inverse Nonsubsampled shear lets Transform. For getting the MRI/SPECT finale fused image the pre-fused image is transformed to RGB color space by integrated with hue and saturate components.

\section{EXPERIMENT RESULTS AND ANALYSIS}

\section{Evaluation Criteria}

To evaluate the performance of the proposed fusion method based on NSST and HIS, four evaluation criteria metrics have been used in this paper. And they are peak signal-to-noise ratio (PSNR) [7], space frequency (SF) [8], information entropy (IE), and edge-base similarity measure $\left(Q^{A B / F}\right)$ [9]. The higher value of the objective criteria is, the better performance of the fused image is.

Peak signal-to-noise ratio (PSNR): PSNR gives the relationship between the fused and the reference image. 


$$
\text { PSNR }=10 \log _{10} \frac{(255)^{2}}{\mathrm{MSE}}
$$

Where the MSE is the mean square error. A higher value of PSNR indicates better quality of the fused image.

Space frequency (SF): represent the definition and the clarity of the image. For given image spatial row frequency $(\mathrm{RF})$ and spatial column frequency $(\mathrm{CF})$, the SF is defined as:

$$
\begin{gathered}
S F=\sqrt{R F^{2}+C F^{2}} \\
R F=\sqrt{\frac{1}{M \times N} \sum_{i=1}^{M} \sum_{j=2}^{N}[F(i, j)-F(i, j-1)]^{2}}
\end{gathered}
$$

And

$$
C F=\sqrt{\frac{1}{M \times N} \sum_{i=1}^{N} \sum_{j=2}^{M}[F(i, j)-F(i, j-1)]^{2}}
$$

The bigger SF is, the better textures of the fused image get.

Information entropy (IE): reflects the amount of information carried by image, bigger entropy means better fusion effect and stronger detail expression performance. It can be described as:

$$
\mathrm{I} E_{A}=\frac{1}{|R|} \sum_{i . j \in R}\left(L_{A}(i, j)\right)^{2} \log \left(L_{A}(i, j)\right)^{2}
$$

Edge-base similarity measure $\left(Q^{A B / F}\right): Q^{A B / F}$ measures the amount of edge information correctly transferred from input source images to the fused image:

$$
\mathrm{Q}^{\mathrm{AB} / \mathrm{F}}=\frac{\sum_{\mathrm{n}=1}^{\mathrm{N}} \sum_{\mathrm{m}=1}^{\mathrm{M}} \mathrm{Q}^{\mathrm{AF}}(\mathrm{n}, \mathrm{m}) \mathrm{W}^{\mathrm{A}}(\mathrm{n}, \mathrm{m})+\mathrm{Q}^{\mathrm{BF}}(\mathrm{n}, \mathrm{m}) \mathrm{W}^{\mathrm{B}}(\mathrm{n}, \mathrm{m})}{\sum_{\mathrm{i}=1}^{\mathrm{N}} \sum_{\mathrm{J}=1}^{\mathrm{M}}\left[\mathrm{W}^{\mathrm{A}}(\mathrm{i}, \mathrm{j})+\mathrm{W}^{\mathrm{B}}(\mathrm{i}, \mathrm{j})\right]}
$$

Where $W^{A}(n, m)$ and $W^{B}(n, m)$ are weights for edge preservation values $Q^{A F}(n, m)$ and $Q^{A F}(n, m)$, respectively The range of $Q^{A B / F}$ is $0 \leq \mathrm{Q}^{\mathrm{AB} / \mathrm{F}} \leq 1$. A higher value of $\mathrm{Q}^{\mathrm{AB} / \mathrm{F}}$ implies that fused image has better edge information.

\section{Experiment Result and Analysis}

To evaluate the performance of the proposed method, four pairs of the experimental image have been implemented. The proposed method is compared with wavelet transform based method [10], PCA based method [11], and NSCT (Nonsubsampled Contour let Transform) based method [12]. The medical brain images that used in this paper can be downloaded from the website:(http://med.harvard.edu/AANLIB/home.html). In this paper, the source images will be decomposed into one low frequency sub-band and several high frequency sub-bands at level $l=3$ and direction $\theta=$ $[2,3,3]$.

The first two groups experiment results of the MRI/SPECT fusion are shown in Fig. 2 (a)-(b). The second two groups experiment results of the MRI/SPET fusion are shown in Fig. 2 (c)-(d).

The objective metrics demonstrate the various quality measurements for different image fusion algorithm. The proposed algorithm is effectively completed multi-input images fusion. Via the evaluation criteria, the performance of the proposed method is shown in table 1, which significantly outperforms the other three methods.

TABLE 1. The fusion quality indexes with different algorithm.

\begin{tabular}{ccccccccccccc}
\hline & & PSNR & SF & IE & $\mathrm{Q}^{\wedge}(\mathrm{AB} / \mathrm{F})$ & & & PSNR & SF & IE & $\mathrm{Q}^{\wedge}(\mathrm{AB} / \mathrm{F})$ \\
\hline & DWT & 67.823 & 17.308 & 4.630 & 59.004 & & DWT & 69.027 & 18.792 & 4.429 & 68.568 \\
pair & PCA & 69.770 & 8.747 & 4.683 & 36.096 & pair & PCA & 71.389 & 19.011 & 4.681 & 53.469 \\
(a) & NSCT & 72.351 & 12.885 & 4.563 & 41.346 & (c) & NSCT & 73.921 & 21.189 & 4.644 & 63.958 \\
& Ours & 74.281 & 20.904 & 4.858 & 69.378 & & Ours & 76.123 & 29.281 & 5.388 & 70.885 \\
& DWT & 61.323 & 18.335 & 4.086 & 56.233 & & DWT & 63.870 & 17.367 & 4.330 & 68.404 \\
pair & PCA & 60.750 & 8.401 & 4.661 & 30.245 & pair & PCA & 65.321 & 20.032 & 4.734 & 48.484 \\
(b) & NSCT & 65.351 & 11.450 & 4.163 & 35.490 & (d) & NSCT & 68.451 & 23.926 & 4.709 & 69.906 \\
& Ours & 70.823 & 18.521 & 4.160 & 57.140 & & Ours & 73.726 & 29.614 & 5.531 & 70.590 \\
\hline
\end{tabular}



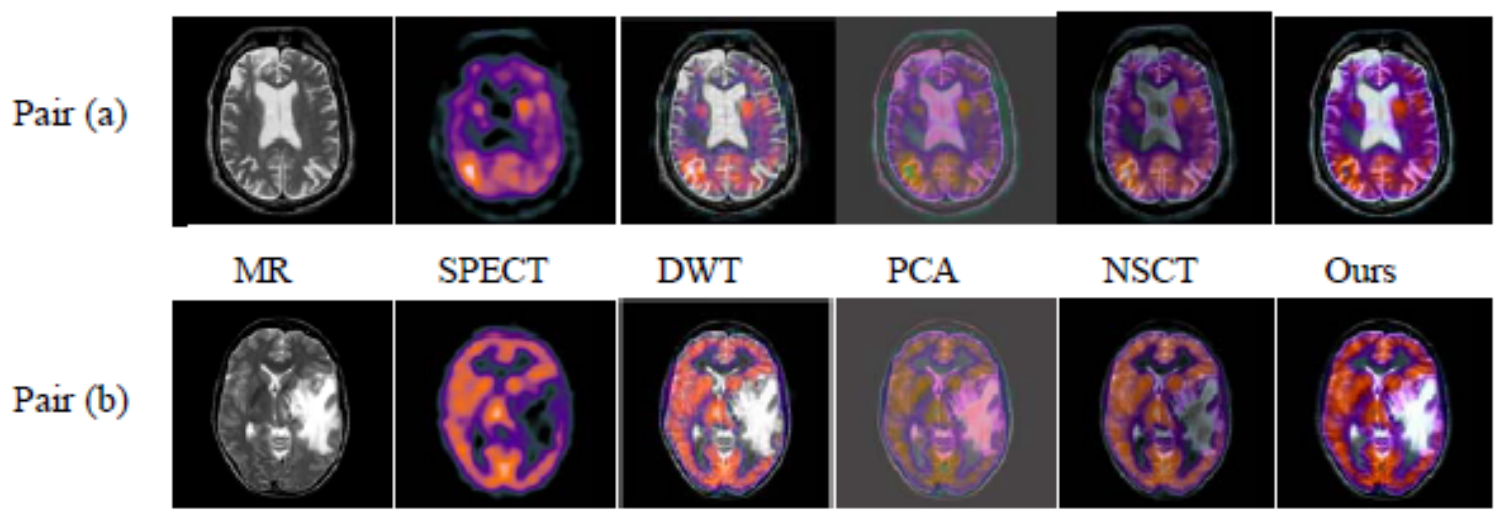

DWT
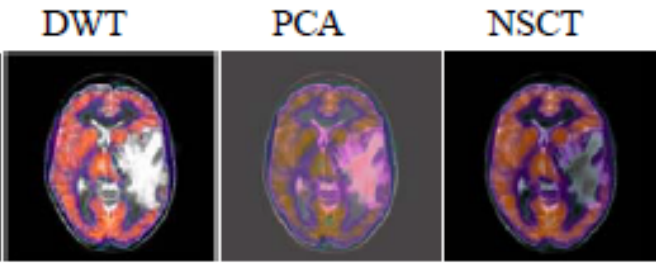

Ours

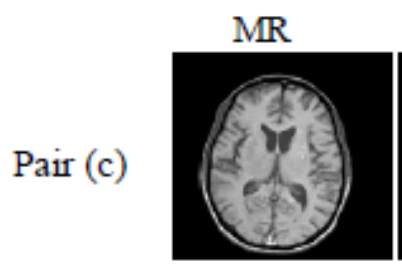

SPECT

DWT

PCA

NSCT
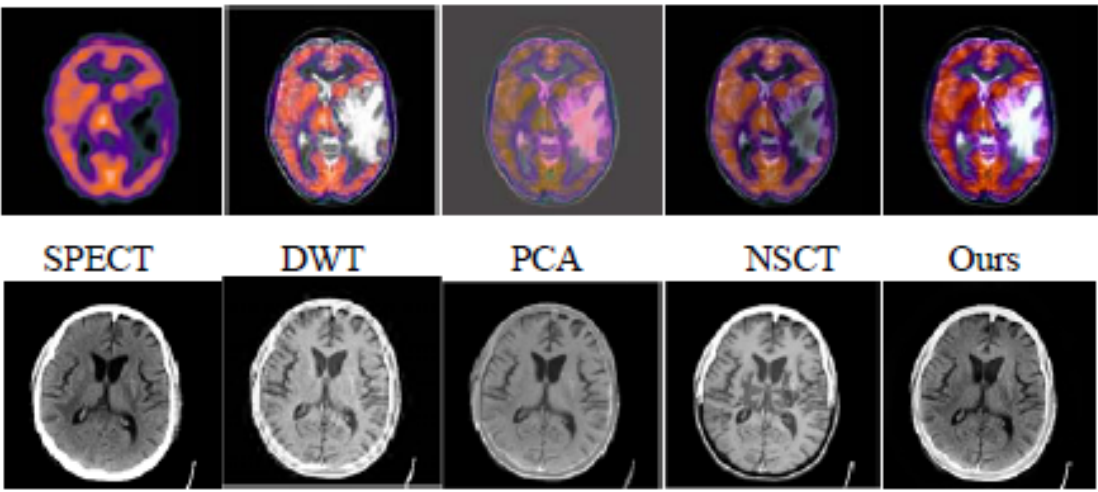

Ours

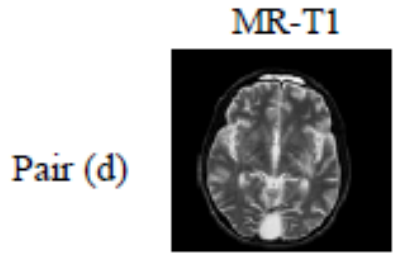

MR-T2

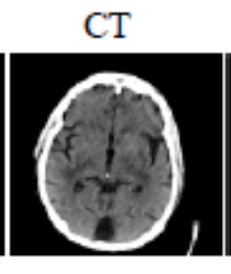

CT
DWT

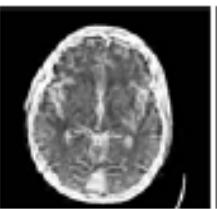

DWT

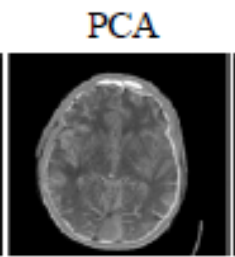

PCA

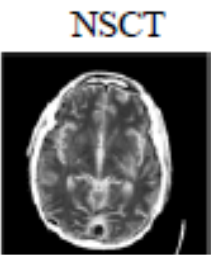

NSCT

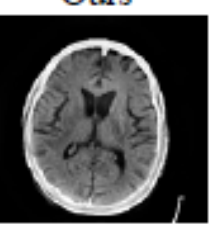

Ours

FIGURE 2. The source brain images and the fused images based on various algorithm.

\section{SUMMARY}

A novel medical image fusion algorithm based on Nonsubsampled shear lets transform is proposed in this paper. In the proposed method, the multi-modal source image is represented in the Nonsubsampled shear lets transform domain. The different fusion rules are applied to the high frequency and the low frequency coefficients. Experiments show that the proposed can well complete multi-modal medical image fusion.

\section{ACKNOWLEDGMENTS}

This work is supported by National Natural Science Foundation of China (11771115) and Youth Foundation of Hebei Province (QN2016169).

\section{REFERENCES}

1. W. Backfired, R. Hand, ET... Image Fusion. Digital(R) Evolution in Radiology. 2001. pp.131-139.

2. Haiku Wang. A New Multiwavelets-Based Approach to Image Fusion. Journal of Mathematical Imaging and Vision. 2004. pp. 177-192.

3. Karl T Dietrich, Yuka Mature, ET. Time series analysis of in vivo cardiac MRI-PET image fusion of the human amniotic mesenchymal stem cell (hams) engraftment. Journal of Cardiovascular Magnetic Resonance. 16Supp:1(2014). Pp.358-359.

4. G Easley, D. labiate, WQ Lim, Sparse directional image representations using the discrete shear let reansfhorm. Appl. Compute. Harmon. Anal. 2008, 25, pp. 25-46. 
5. To, T.-M., Su, S.-C., Shy, H.-C., and Huang, P. S.A new look at HIS-like image fusion methods. Information fusion 2001,2(3) pp.177-186.

6. T.M. Cover, J.A. Thomas, Elements of information theory, John Wiley and Sons, Inc., New York, NY,1991.

7. Horseman. \& Zion, D. Image quality metrics: PSNR vs. SSIM. 2010 20th international conference on pattern recognition(ICPR), 2010, pp. 2366-2369.

8. A.M. Eskicioglu, P.S. Fisher, Image quality measures and their performance, IEEE Trans. Common., 1995, 43(12) pp. 2959-2965.

9. Xydis, C. S., and Petro Vic. V., Objective image fusion performance measure. Electron. Let. 2000, 36(4) pp. 308-309.

10. Jionghua Tang, Xu Wang, ET. A Multimodality Medical Image Fusion Algorithm Based on wavelets Transform. ICSI2010. Beijing, 2010.6.12, pp.627-633.

11. MJ Liu, YS Dai, J Zhang, et al., PCA-based sea-ice image fusion of optical data by HIS transform and SAR data by wavelet transform. Act Oceana. Sin., 2015, vol. 34, No.3, pp.59-67.

12. V Bhatia, A Srivastava, A Mino, et al., NSCT based multispectral medical image fusion model. IEEE Instrumentation and Measurement Society $2016 \mathrm{pp} .1-5$.

13. http://med.harvard.edu/AANLIB/home.html. 